\title{
XR for XR - Context Craft: An extended reality platform that transforms local environments through play
}

\author{
Carl H. Smith \\ Learning Technology Research Centre \\ Ravensbourne University London \\ 6 Penrose Way, Greenwich Peninsula, \\ London, UK \\ c.smith@rave.ac.uk
}

\author{
Jay F. Cousins \\ Circus of Seeds \\ Corner house, North End Road, Abu \\ Mina Street \\ Dahab, Egypt \\ Jayfcousins@gmail.com
}

\begin{abstract}
The aim of Context Craft is to develop a platform that shrinks the gap between imagination and reality. Players become crafters of their own context - hacking their reality for the benefit of themselves and their communities. Imagine if you could take what you made in Lego or Minecraft and make it in reality with household tools and some globally accessible waste (such as soda bottles, water bottles, sticks for core framework, plastic bags, fabric, cans etc.) and "mine" reality in order to transform it via polygon structures that can be used to make anything from furniture, to bike trailers to shelters and community buildings. XR (Cross or Extended Reality) is a recent, controversial, umbrella term that attempts to simplify the understanding and adoption of the full range of immersive technologies that generate new forms of reality, recognising that in the future we will see people interacting with the virtual world and real world in seamless and continuous ways, not bound by delineations of experience between VR, AR, and MR. Recently, another organisation Extinction Rebellion also adopted the acronym XR. This serendipity led to the questioning how can these XR technologies relate to our Extinction Rebellion (XR)?
\end{abstract}

Extended reality (XR). Cross reality. Extinction Rebellion. Upcycling. Environment. Context. Ecosystem. Social ecology.

\section{INTRODUCTION}

We've evolved to be particular ways in particular settings, context, context, context (Sapolsky 2017)

Context Engineering (CE) focuses on manipulating and creating contexts directly. Context Engineering (Smith 2014) explores how the possibilities of analogue (physical) and the digital (virtual) worlds might relate and merge, through the creation of new tools and practices. We are now in an era where context adaption and augmentation is central to mitigate against negative behaviour change caused by poorly designed technologies. According to (Cheok 2012), advances in technology are moving us from an era of 'information communication' to 'experience communication'. The context engineering of hybrid space involves the fundamental augmentation of perception giving us control over our contexts and senses, allowing us to adjust them in real time. Context engineering is beginning to create a new economy where our own perception becomes the 'content'.

Humans adapt to contexts, and we adapt contexts to us. Many of the present contexts to which we have adapted reward behaviours and ideas which are harmful to ourselves, society and our life sustaining ecosystem. We are incentivised to extract value and externalise costs. This encourages scarcity, exploitation, dumping and waste.

Extinction Rebellion is a call for systemic change through a reaction to the destructive legacy of the contexts, which are leading to our collective extinction (Basden 2019). To achieve any real level of agency or free will in the world we must develop a contextual awareness and literacy - for to change ourselves we must learn to adapt the contexts of our existence. The core question is how do we use $\mathrm{XR}$ technologies to shift from reactive behaviours to creative ones and bring a new reality into being? 
However reaction alone is insufficient as we are interdependent with these toxic contexts. The hand that poisons also feeds. We need systemic change. We require new relationships, structures and systems that sustain us and our environment, and we have to build them fast.

We need to adapt our existing contexts whilst creating new contexts to which we can migrate. Parallel to this, we must increase our common capacities for adaptation, as great changes are coming, we must adapt fast to thrive and survive. This is the mission of Context Craft.

\section{WHY AN XR GAME?}

The opposite of play isn't work, it's depression. (McGonigal 2010)

The emotional burden represented by the reality of the death of our ecosystem and our complicity in it leads to denial or nihilism. This prevents us from acting as we need to, as helplessness and apathy are not constructive emotional states. They are states of withdrawal and reluctant acceptance.

An Extended Reality (XR) game provides us with the means to explore what a new reality could look like through play, and to learn how to adapt our existing reality to better meet our collective needs and those of our life sustaining systems. It allows the suspension of our existing norms and systems and allows us to face up to catastrophic scenarios in a non-threatening way. The game allows us to prototype and experience new systems of existence and share them with the world.

Our goal is ambitious: Global systemic transformation through extended reality play. Context Craft increases our shared abilities to adapt, and increase our chances of thriving in a fast changing world.

\section{STORIES AS SHARED CONTEXTS}

An ancient metaphor: thought is a thread, and the raconteur is a spinner of yarns -- but the true storyteller, the poet, is a weaver. The scribes made this old and audible abstraction into a new and visible fact. After long practice, their work took on such an even, flexible texture that they called the written page a textus, which means cloth (Bringhurst 1992).

Context is literally "to weave together". Our shared myths and stories create a common cloth and shared social objects that allow us to engage with the shared context of our common existence. However our stories have long been ones of separation and hierarchy. The cloth is torn. Even our words and metaphors have been cleaved of meaning. Allowing for their exploitation and distortion.

These tales are so embedded in our collective psyche that some believe there are no alternatives; that choices are somehow static and baked into our culture, and we are perpetually doomed to choose the "least harmful path". Our narratives of humanity and society, largely spread through mediated myths, amplified by power - that the only options we have are those that the powerful provide. "It's the way things are". "Accept it" We do not accept the choices presented. We seek to create new choices for a better quality of existence. We must collect the disconnected threads of our shared existence and weave a new narrative. Context Craft provides us with a platform and an invitation to reweave a common cloth.

\section{A NEW STORY, A NEW REALITY}

Until society can be reclaimed by an undivided humanity that will use its collective wisdom, cultural achievements, technological innovations, scientific knowledge, and innate creativity for its own benefit and for that of the natural world, all ecological problems will have their roots in social problems. (Bookchin 1989)

In Context Craft we provide space for exploration of shared and alternative narratives, around which players may add their own details and explore what possibilities exist to help them create a new reality. This narrative framework starts small and simple, as it should be accessible from ages 8 onwards (with parental support and supervision for life sized builds).

The game prioritises learning through experience, using material structures as the initial path to learning to build material literacy and contextual literacy (the ability to read/right your environments - starting with your home spaces and expanding to your local and ultimately global spaces).

Other literacies and learning paths will be expanded upon as the game progresses to include; emotional literacy, environmental literacy, narrative literacy, power literacy among others.

Most important of all is to become attention literate - aware of awareness, where attention is placed and how it is directed, in order to connect with reality before we abstract it. To connect words to their metaphors; to learn through awareness and curiosity. 


\section{A.R.C.S}

One of the objectives of the game is the creation and replication of A.R.C.S (Cousins 2019) Accessible Regenerative Common Systems to provide Universal Basic Services for all the beings of the Earth. Players are introduced to the A.R.C.S and how to build early examples through playing the game. At higher levels they are encouraged to conceive and grow their own. An example of A.R.C.S is the "reality construction kit", which is the first learning path offered by the game.

The reality construction kit takes the basic engineering principles learned by playing with the game's analogue toy structures or their digital counterparts, and allows the player to build life sized stable structures and systems from waste material. Stability of builds is measured through peer testing, which should be continuously undertaken by the community.

Players must progress by building and testing simple low risk structures in the initial phases of the game (starting with toys scale objects to understand design basics). The designs themselves can also be assessed by ML algorithms, and with modelling for breaking of critical nodes to assess risk of builds in advance of construction.

It is Accessible because the barrier to creation is low - materials are free and globally distributed.

It is Regenerative because it is easy to repair and replicate.

It is Common because anyone can build it and all knowledge is shared freely.

It is a System that produces useful structures around which other essential systems can be built.

Both initial game elements and outcomes can be described as A.R.C.S as they fulfil the above criteria.

\section{INITIAL GAME ELEMENTS}

Initial game elements create a wealth of opportunities for adapting your context; from prototyping tools to physically shaping your surroundings with ease. They can be used separately and in combination.

\subsection{Prototape}

Prototape functions like reusable duct tape strong, waterproof and versatile.

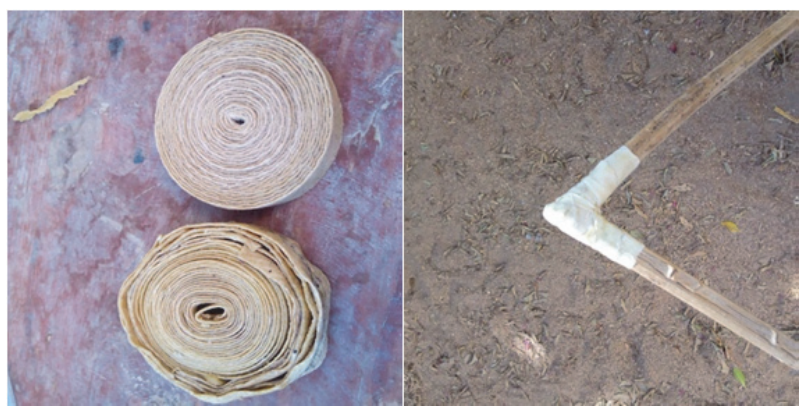

Figure 1: Prototape rolls, Prototape binding sticks.

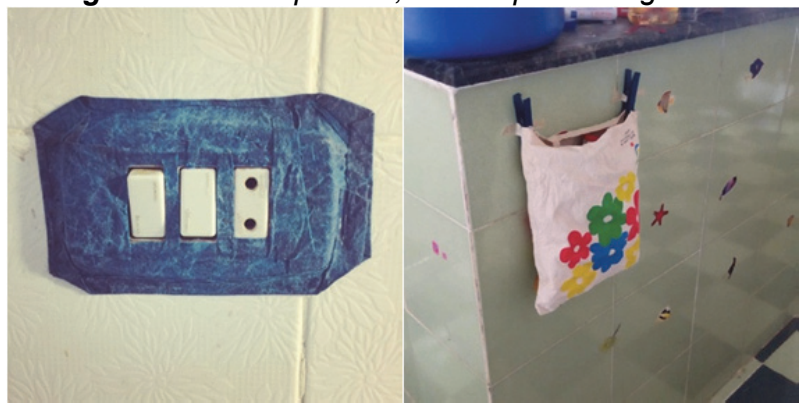

Figure 2: Coloured Prototape used as light switch cover, Bag stuck to wall with Prototape.

\subsection{Sticky String}

Sticky String is a DIY version of Wikistix and similar kids toys. Made from Beeswax and string it functions in much the same way as Prototape, just with a smaller width. Both Sticky String and Prototape, create opportunities for customisation of space, and creation of real world AR markers and symbols created by players. They leave no residue on walls and can be used to bear modest loads.

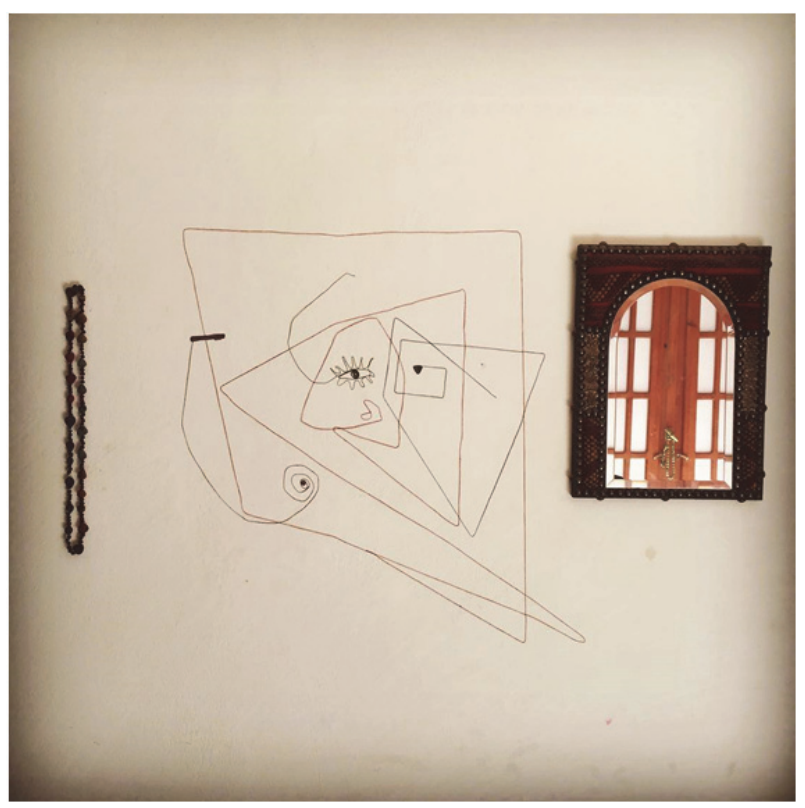

Figure 3: Mural from Sticky String. 


\subsection{Construction kit}

The construction kit uses small pieces of Prototape and Sticky String with coloured sticks of differing length to construct a host of 3D forms. By using triangles as the base structure we ensure that creations are strong and can be played with without failure (or easily repaired should failure occur).

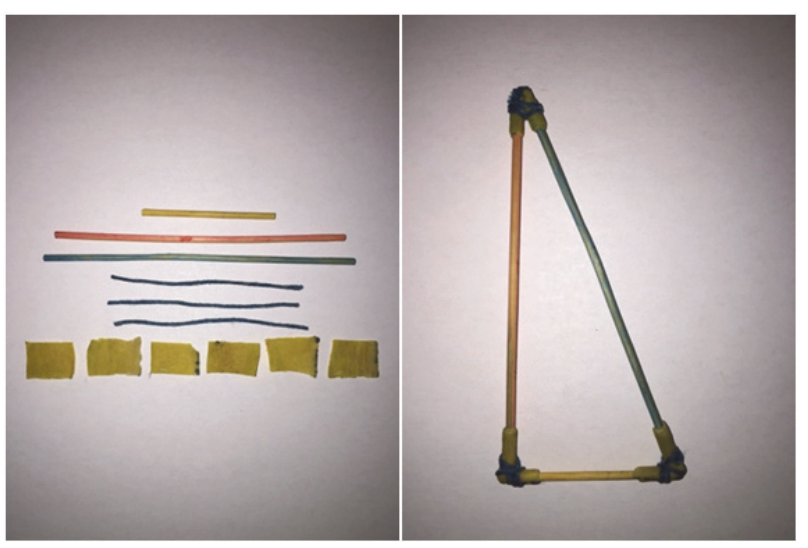

Figure 4: Construction pieces and "seed" structure.

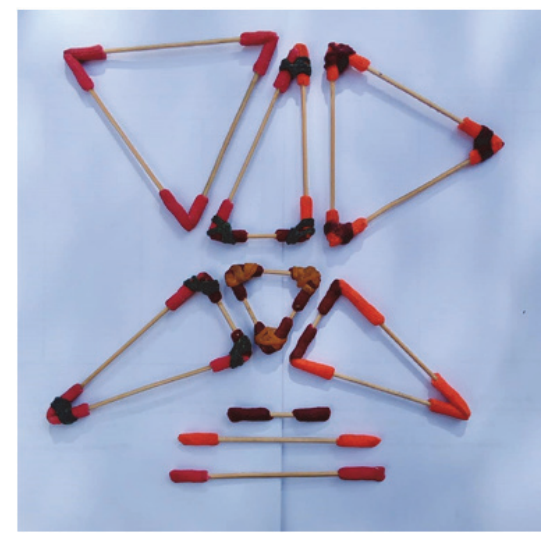

Figure 5: Variations of "seed structures" (previous prototype).

From these initial structures, an infinite number of forms maybe created, and combined with additional materials to increase uses. Players are encouraged to use "waste" materials in their designs.

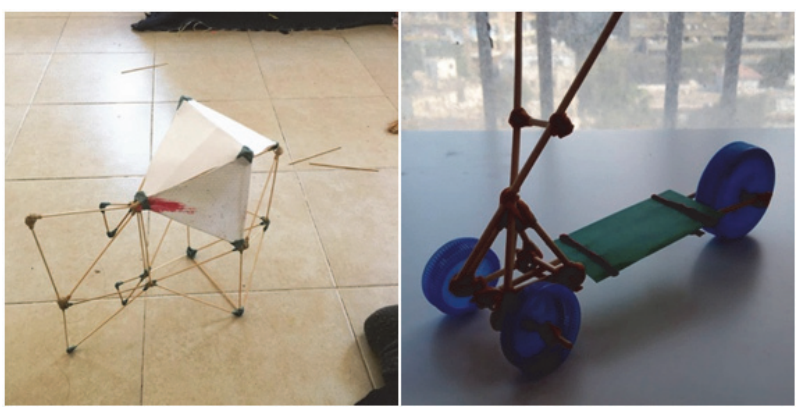

Figure 6: Experiments connecting materials
This addition of household materials allows for the creation of moving parts. Future iterations will also allow for electronic components (Prototape can be used in place of solder to connect wires).

\subsection{Reusable cable ties}

Reusable cable ties are made from soda bottles, transforming an environmental hazard into a useful resource.

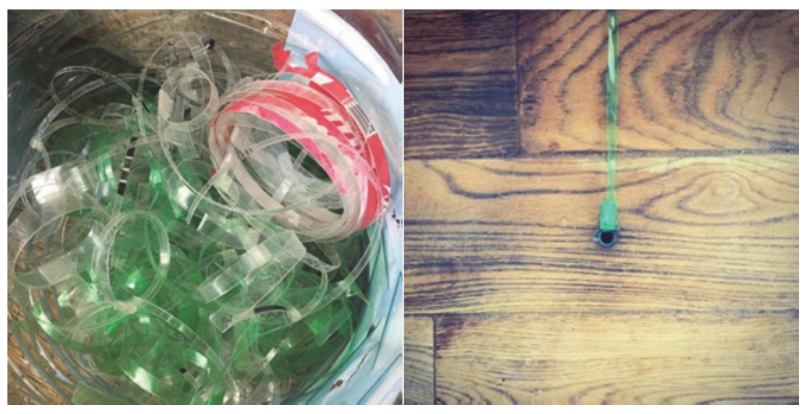

Figure 7: Reusable cable ties from soda bottle cord.

They can be easily made with a pair of scissors, or more complex processes and tools can be built for efficient production. The first step is the production of bottle cord (Егоров 2014).

\subsection{Reality construction kit}

Using the cable ties, combined with sticks, and plastic bottle joins - heating bottle plastic shrinks material allowing for strong joins to be formed (Pedros 2016). Combining these processes with space frame design principles (Bell 1898). Allows for the construction of strong lightweight structures, which can be first explored and prototyped with the construction toy.

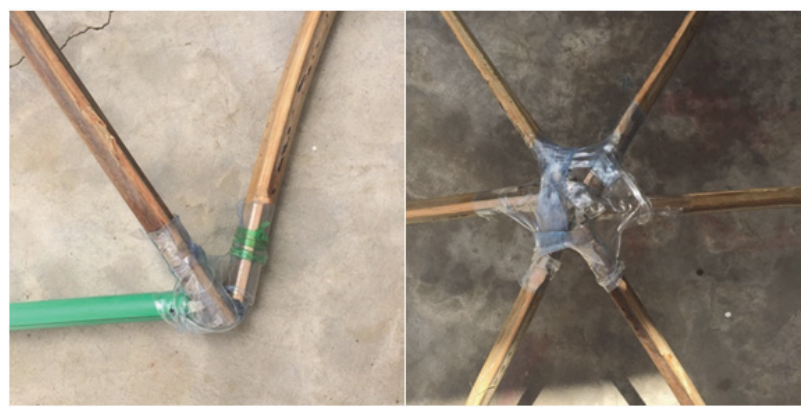

Figure 8: Recycled plastic joints.

This combination of existing methods, and new adaptations, creates the opportunity for a wide range of possible useful structures, a scaffold around which more systems may be built. 


\section{OUTCOMES FROM EXISTING GAMEPLAY}

Through play testing the game, initial players have enabled the following ideas to become real.

\subsection{Multiple iterations of bike trailers}

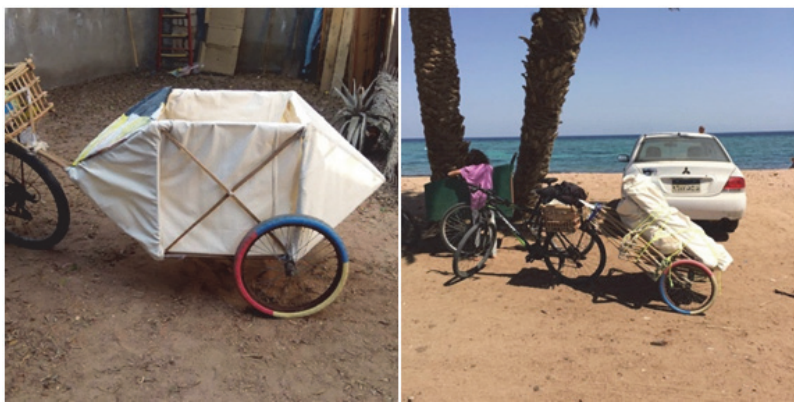

Figure 9: Bike Trailer Container, and Expandable Frame Models.

\subsection{Multiple household furniture designs}

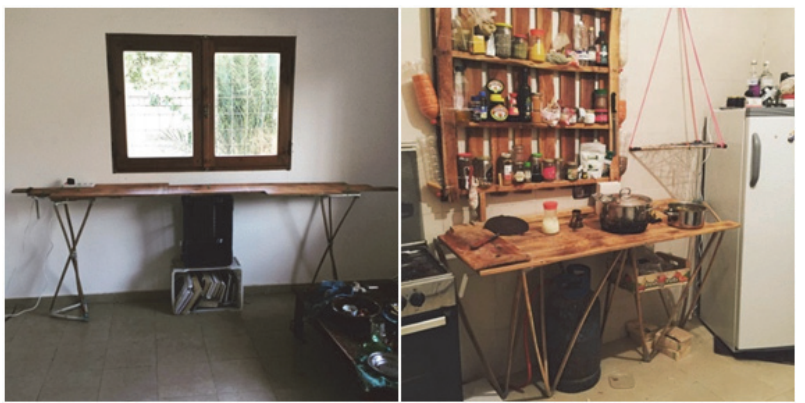

Figure 10: Standing desk pillars, and kitchen worktop frame.

\subsection{Kids play dome}

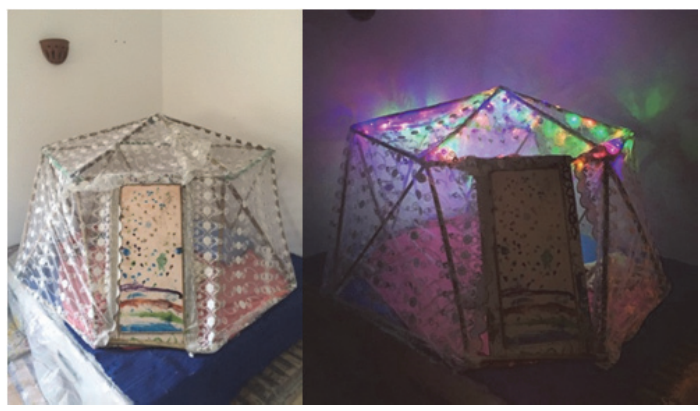

Figure 11: Kids play dome (now a slumber tent).

\subsection{Co-working space furniture}

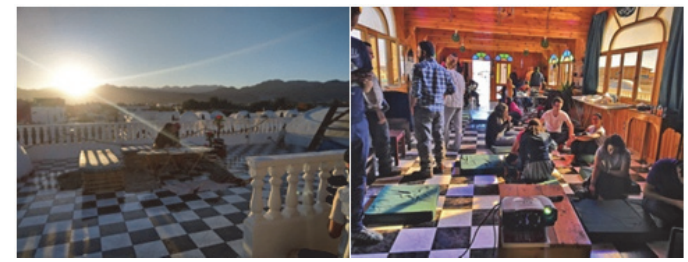

Figure 12: Outdoor and indoor furniture creations at Alchemy Hub.
In addition to this, they allow for the exploration of numerous other possibilities not yet constructed.

\subsection{Folding dome structure}

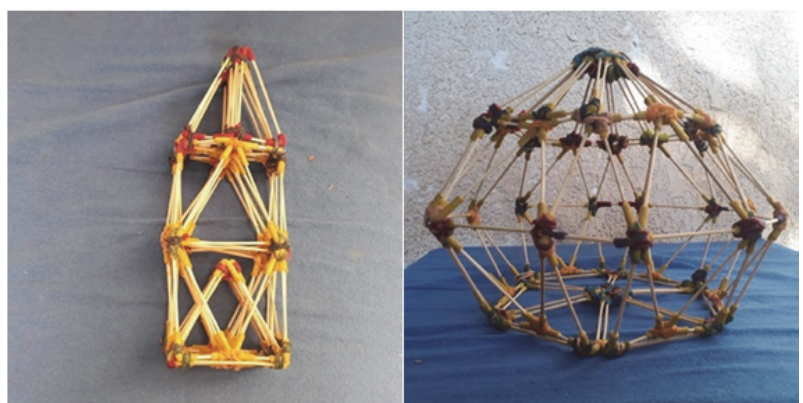

Figure 13: Folding dome design, real version $4.8 \mathrm{~m}$ diameter at base.

In addition to the structures already built the secondary effects of play have resulted in increased confidence, decreased fear of future due to increase in adaptive capabilities, and weight loss due to frequent movement and activity.

\section{XR OPPORTUNITIES}

XR (Cross or Extended Reality) covers the full range of immersive technologies that generate new forms of reality. These technologies include Mobile Learning (ML), Virtual Reality (VR), Augmented Reality (AR) and Mixed Reality (MR). Recently another organisation, Extinction Rebellion also adopted the acronym XR. This prompts the question how can $\mathrm{XR}$ technologies help us with our Extinction Rebellion (XR)? How can we use these technologies to help us transform the world?

Initial development has focussed on shrinking the gap between the accessible material world and the adjacent possible world.

The digital realm and the extended realities it enables, act as a medium, which can allow for better communication of the adjacent possible. It offers new opportunities to reconnect with our metaphors of existence and bring the read/write behaviours the web has nurtured and enabled into our common and material spaces.

The opportunities listed are by no means exhaustive - as co-created real world building leads us into infinite possibilities for helpful technological tools. The elements described below represent our first explorations, and initial goals for $\mathrm{XR}$ interaction.

\subsection{Ethical compass}

The "ethical compass" provides a minimum viable moral framework for gameplay, guiding the actions 
and decisions of players. It can be framed as two questions:

1. In which contexts is this harmful?

2. In which contexts is this useful?

By viewing an item through the ethical compass, information on how the item has already caused harm, and its potential for further harm can be understood. In addition to this, players can see how the item can be used for collective benefit (and add their own discoveries to the object). From these uses missions are presented to players, accepting missions leads players on a learning journey to either move or transform the item in question.

By viewing an item through the ethical compass, information on how the item has already caused harm, and its potential for further harm can be understood. In addition to this, players can see how the item can be used for collective benefit (and add their own discoveries to the object). From these uses missions are presented to players, accepting missions leads players on a learning journey to either move or transform the item in question.

\subsection{AR object scanning}

Scanning and geo-tagging creations made either with construction toy, or larger kit. Patterns on sticks will denote length and allow for easy data conversion.

\subsection{AR Object placement, modification, and learning paths}

Using AR 3D modelling tools to place player constructed virtual objects in spaces, and create new forms by using phone to drag and drop forms in space. Objects can also be tagged by context for ease of discovery - e.g. Road shows a bike trailer, kitchen shows a dryer or shelf. Forms can be selected as missions for players to discover and build in reality.

\subsection{DIY Holoportal}

Creating a DIY Holoportal (dome with 360 degree cameras and scanning) to allow for more detailed object scanning and human movement scanning. This could be combined with Mixed Reality (MR) to allow players to embody the movements of artisans in real time - matching their movements to those of the original performer that they see in real time.

\subsection{Marketplace for open source Ideas}

Open source usually provides the greatest opportunities for any given idea, however value rarely flows directly back to the creator (which not only reduces the incentive to work, but also impacts the quality of the work itself as there is no feedback loop regarding the works fitness for purpose). As a result Context Craft functions as a point of discovery for useful ideas, which themselves have a material cost and cost of time to acquire, there is the opportunity for both inventors and makers to be rewarded for their work.

\subsection{Al for structural testing and evaluation}

Proposed structures and forms can be stress tested using the models using $\mathrm{Al} / \mathrm{ML}$ techniques.

\subsection{Al representation of non-human players}

Whilst human players can create missions, other beings that also serve the Earth should also be presented in game in order that their Value contribution is not ignored.

\subsection{Documenting value at point of service}

Value or rewards are created in Context Craft by serving other beings. When a need is communicated value is "mined"; when it is served currency is "minted". Proof of service can be easily created by two players taking a selfie along with evidence that service has taken place. Both players then communicate at same time to create proof of service.

\subsection{A/B testing alternate patterns of existence and supporting technologies}

The Game provides space and a marketplace for the testing, prototyping and distribution of Alternative Technologies and Systems that are useful to the social ecology.

\subsection{Niantic platform}

We are using the Niantic platform for the following reasons:

Tested capacity to deliver an upscaled global location based AR experience to over 147 mil users (May 2018). Implies good quality and reliability of the SDK and platform.

The new features announced will allow smart phone capacity is something close to a mixed reality device:

AR with Occlusion Technology provides superior spatial understanding resulting in consistent object placement raising precision and reliability of the virtual constructs as well as greater visual integration within the AR composition with it's realtime occlusion, something close to what MR headsets are capable of.

The acquisition of Escher Reality and Matrix Mill announce Object Recognition (via computer vision 
+ machine learning) means we will be able to get more organic shapes into the experience via the phone, have more accurate measurements from the real world, bringing Context Craft elements very close to the ones in reality, making it a practical, useful, realistic tool.

\section{GAME AS LIVEABLE REALITY}

For the game to become a liveable reality it must provide the services required for a good quality of life with lower energy (cost) requirements and greater rewards that our existing reality under the existing toxic legacy contexts (state/amoral market/violence backed authority). It should be noted that historical responses to alternative systems (war, coup, sponsored insurgence, acquisition, or appropriation) usually result in the collapse or perversion of the alternative before it can be proved viable.

For the game to succeed therefore it must offer not just an alternative to the existing system, but prove itself useful to those who possess existing power (or at least a sufficient percentage to ensure it's protection), and those who are damaged daily by these toxic legacy contexts.

The ideal situation is a parallel social ecology that incentivises resource transfer and asset migration.

To this end profits from the game, beyond those required by the game providers and developers will be invested in common land, A.R.C.S and their production to better serve players.

These community assets will then provide priority access to players who serve the most other beings globally (note: this includes non human players for example forests).

\section{GAME AS SELF-MANIFESTING SYMBIOTE}

As a part of the game play, players are encouraged to replicate the physical game and larger reality construction kit, as well as recruiting more players to the game itself. As the game accesses more players as "embodiments" to shape the world to it's "will" (to serve all beings and nurture their growth), it can be understood as a self replicating symbiote which will ultimately incorporate both artificial intelligence, humans and other planetary life sustaining organisms. This will be expanded at deeper levels of the story, and offers further opportunities for exploration of spiritual, technological and intellectual development of the players and the culture it nurtures.

The game should function as a positive form of Roko's Basilisk (Roko, 2012) focused on present day incentives rather than retroactive penalties to bring a symbiotic intelligence and life supporting infrastructures into being.

\section{CONCLUSIONS}

The following are key findings from our investigation:

1. The experience of making things with hands is a skill in great decline. Even the maker movement is full of people who lack basic material literacy instead favouring digital tools like laser cutters and 3D printers.

2. Key to be device agnostic in order to be as device diagnostic as possible - from smart phones to MR devices (ML + HoloLens)

3. Use Al to evaluate structures - structural capacity - using Machine Learning.

4. Regulatory policy: does your construction passing health and safety standards in your location?

5. The system provides associated carbon costs / actual costs of any given materials.

6. Material literacy

7. Reward system for building on other peoples designs

8. Highlighting issues in the $3 d$ printing community: use the system to decide if you need to utilise $3 \mathrm{~d}$ printing as well

9. Find solutions of maximum utility and benefit

10. Al can help us to see different solutions are available that we did not think of.

In addition, the following are the main early outcomes of this work:

1. Accelerated prototyping processes, increased availability of ideas and lower barriers to making them happen.

2. Players are also incentivised to increase personal and community capabilities.

3. Changing the player's perspective of the many forms of waste that are available for transformation.

4. Increase of adaptive capacity i.e. adapt the environment to better meet our needs.

\section{REFERENCES}

Basden S. (2019) Extinction Rebellion isn't about the climate, Medium. http://tinyurl.com/y2npjvdx (retrieved 12 April 2019).

Bell, A. (1898) Space frame principles, discussed in Wikipedia entry. https://en.wikipedia.org/wiki/Space frame (retrieved 12 April 2019).

Bookchin, M. (1989) Remaking Society. Black Rose Books. 
Bringhurst, R. (1992) The Elements of Typographic Style. Hartley \& Marks Publishers.

Cheok, A. (2012) ICALT2012 keynote talk. http://adriancheok.info/category-speech/icalt2012keynote-talk-multi-modal-sensory-human (retrieved 12 April 2019).

Cousins J. (2019) The Game Plays Me, Medium. https://medium.com/@jaycousins/the-game-playsme-f3bae1b2863c (retrieved 12 April 2019).

Егоров A. (2014) The STRAND version 2.0 Application of the rope out of the bottle. https://www.youtube.com/watch?v=hQeeJEpBYsg (retrieved 12 April 2019)

Kaufmann S. (2008) Reinventing the Sacred. Basic Books.
McGonigal, J. (2010) Reality is Broken. Penguin.

Pedros M. (2016) Micaella Pedros [online portfolio]. https://www.micaellapedros.com (retrieved 12 April 2019).

Roko (2012) Roko's basilisk, Rational Wiki entry. https://rationalwiki.org/wiki/Roko\%27s basilisk (retrieved 12 April 2019).

Sapolsky R. (2017) Behave: The Biology of Humans at Our Best and Worst. Penguin Press.

Smith, C. (2014) Context Engineering Hybrid Spaces for Perceptual Augmentation. Electronic Visualisation and the Arts (EVA 2014), British Computer Society, London. 